\title{
An Approach to Distinguish between Plasticity and Non-random Distributions of Behavioral Types Along Urban Gradients in a Wild Passerine Bird
}

\author{
Philipp Sprau ${ }^{1 *}$ and Niels J. Dingemanse ${ }^{1,2}$ \\ ${ }^{1}$ Behavioral Ecology, Department of Biology, Ludwig-Maximilians University of Munich, Munich, Germany, ${ }^{2}$ Research Group \\ Evolutionary Ecology of Variation, Max Planck Institute for Ornithology, Seewiesen, Germany
}

The impact of urbanization has been widely studied in the context of species diversity and life history evolution. Behavioral adaptation, by contrast, remains poorly understood because empirical studies rarely investigate the relative importance of two key mechanisms: plastic responses vs. non-random distributions of behavioral types. We propose here an approach that enables the simultaneous estimation of the respective roles of these distinct mechanisms. We investigated why risky behaviors

OPEN ACCESS

Edited by:

Caroline Isaksson

Lund University, Sweden

Reviewed by:

Wayne C. Zipperer,

USDA Forest Service, United States

Geoffrey M. While,

University of Tasmania, Australia

${ }^{*}$ Correspondence:

Philipp Sprau

philippsprau@gmx.de

Specialty section:

This article was submitted to

Urban Ecology,

a section of the journal

Frontiers in Ecology and Evolution

Received: 30 March 2017

Accepted: 24 July 2017

Published: 08 August 2017

Citation:

Sprau P and Dingemanse NJ (2017)

An Approach to Distinguish between

Plasticity and Non-random Distributions of Behavioral Types

Along Urban Gradients in a Wild

Passerine Bird. Front. Ecol. Evol. 5:92.

doi: 10.3389/fevo.2017.00092 are often associated with urbanization, using an urban nest box population of great tits (Parus major) as a study system. We simultaneously and repeatedly quantified individual behavior (aggression and flight initiation distance) as well as environmental factors characterizing level of urbanization (numbers of pedestrians, cars and cyclists). This enabled us to statistically distinguish plastic responses from patterns of non-random distributions of behavioral types. Data analyses revealed that individuals did not plastically adjust their behavior to the level of urbanization. Behavioral types were instead non-randomly distributed: bold birds occurred more frequently in areas with more cars and fewer pedestrians while shy individuals were predominantly found in areas with fewer cars and more pedestrians. These novel findings imply a major role for behavioral types in the evolutionary ecology of urban environments and call for the full integration of among- and within-individual variation in urban ecological studies.

Keywords: among-individual variation, phenotypic plasticity, personality, urbanization, great tit, within-individual variation

\section{INTRODUCTION}

Urbanization represents one of the fastest environmental changes of our times (United Nations, Department of Economic and Social Affairs, and Population Division, 2017) and poses novel challenges to wildlife (Sih et al., 2011). Urban habitats are characterized by a loss of natural resources (e.g., food) and increased anthropogenic levels of disturbance (e.g., pedestrian and vehicular traffic, and industrial noise). There is a growing body of literature describing how animal populations are affected by urbanization (Marzluff, 2008). In birds, for instance, breeding densities are often higher in urban compared to natural habitats (Beissinger and Osborne, 1982). Furthermore, several studies have reported advanced lay dates, smaller clutch sizes and decreased 
offspring production in urban compared to rural habitats (reviewed in Chamberlain et al., 2009). More recently, research has focussed on behavioral modifications in urban wildlife (Lowry et al., 2013). Yet, the mechanisms responsible for associations between behavior and level of urbanization remain largely unknown.

Phenotypic and environmental variation are often associated (Stearns, 1989). Relationships between phenotypes and environments can be caused by various distinct mechanisms such as phenotypic plasticity, differential settlement, and/or the selective (dis)appearance of distinct types of individuals (Dingemanse et al., 2010). Several studies have demonstrated behavioral plasticity in response to urbanization (Lefebvre, 1995; Seferta et al., 2001; Bouchard et al., 2007; Levey et al., 2009). Song birds such as nightingales (Luscinia megarhynchos) and great tits (Parus major) have been shown to adjust song amplitude (Brumm, 2004) and frequency (Slabbekoorn and den Boer-Visser, 2006) to background noise. Phenotypes, however, also vary among individuals. Repeatable differences among individuals have been demonstrated for a variety of behaviors such as aggressiveness, boldness and exploration (Bell et al., 2009; Garamszegi et al., 2012; Holtmann et al., 2017). These particular types of behavioral traits have recently also been explicitly linked to urbanization. For instance, urban male song sparrows (Melospiza melodia) are bolder and more territorial than their rural conspecifics (Evans et al., 2010). Boldness reflects the tendency of an individual to take risks (Wilson et al., 1994; Réale et al., 2007). Accordingly, bold individuals are often considered to cope better with risky and novel situations and may therefore be favored in highly disturbed areas such as cities.

Importantly, behavioral traits vary simultaneously among and within individuals, due to individual variation (aka "personality") and plasticity (Dingemanse et al., 2010). Therefore, associations between behavior and urbanization can similarly originate from multiple processes. This can be usefully illustrated by viewing behavior in terms of reaction norms (Nussey et al., 2007; Dingemanse et al., 2010), where each individual is characterized by a reaction norm intercept (representing its average behavior in the average environmental condition, or behavioral "type"), and a reaction norm slope, representing its level of response (plasticity) to changes in urbanization (Figure 1). Viewing behavior in this way clarifies that the relationship between behavior and urbanization is the result of the combined effects of phenotypic plasticity and non-random distributions of behavioral types over urban gradients. Previous studies have not distinguished between these two mechanisms in
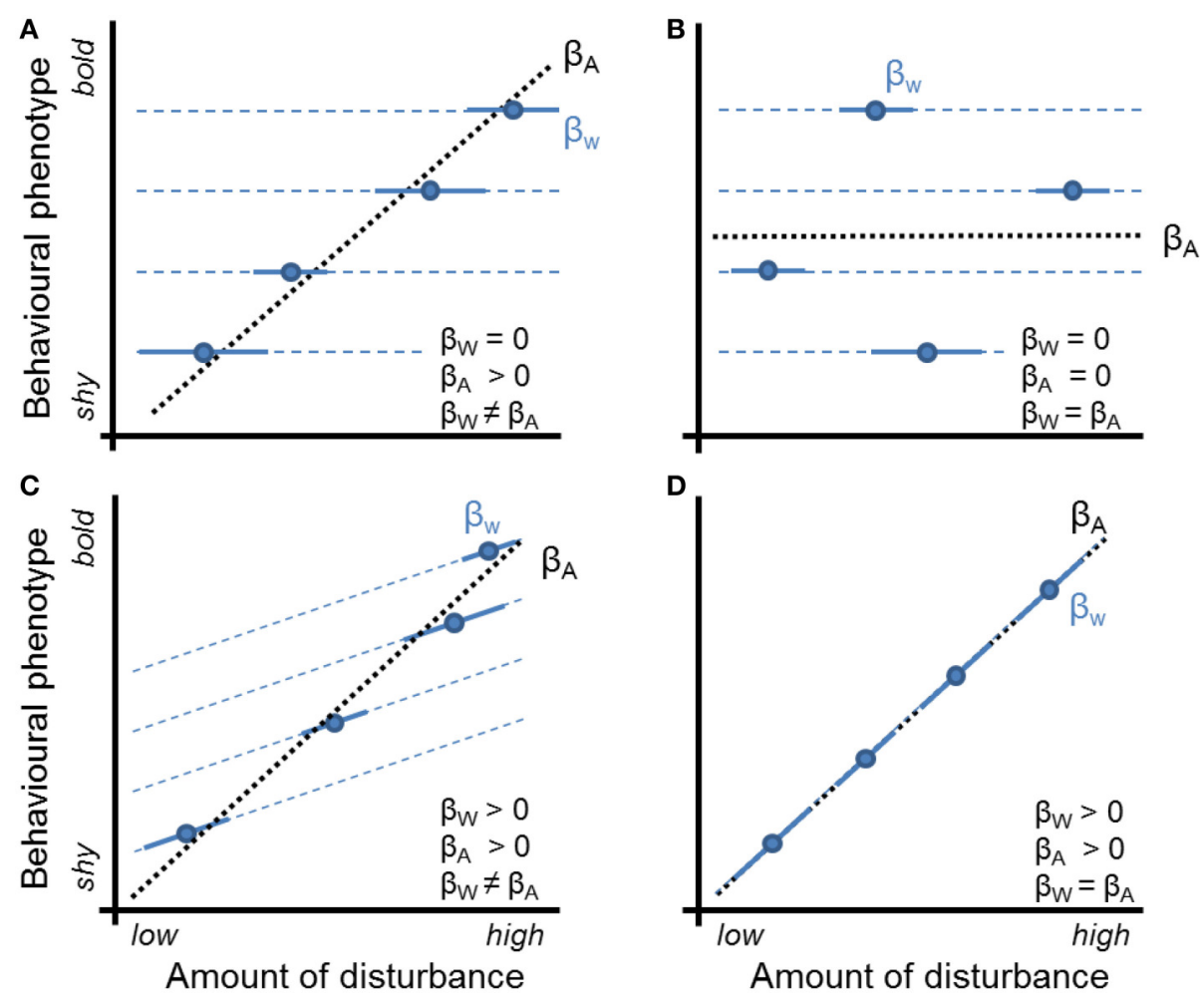

FIGURE 1 | Behavioral reaction norm plots, providing examples of possible relationships between anti-predator boldness (an exemplary behavioral phenotype) and an environmental gradient (e.g., amount of disturbance) among- vs. within-individuals. Each dot represents the average phenotype of an individual and each solid blue line represents its behavioral reaction norm over the range of environmental conditions ( $\mathrm{x}$-axis) that it occurred observationally. Among-individual variation in intercepts represents "personality variation" (sensu Dingemanse et al., 2010). Non-zero slopes represent within-individual plasticity (i.e., the observed relationship between y and $\mathrm{x}$ within the individual: $\beta \mathrm{W}$, blue lines). In contrast, blue dashed lines represent the hypothesized phenotype of each individual outside the range of environments in which it occurred observationally. The thick black dashed line represents the relationship between each individual's average behavior and average environment ( $\left.\beta_{A}\right)$. Plots (A-D) show different scenarios for how behavior and the environment might be associated within and between individuals as detailed in the Introduction. 
empirical demonstrations of relationships between behavior and urbanization.

We conceive four scenarios for how behavior and level of urbanization may be associated, though we recognize that further scenarios could be proposed. In the first scenario, individuals are non-randomly distributed over an urban gradient with respect to their reaction norm intercept (i.e., average behavior, or behavioral type) while they simultaneously do not respond plastically to changes in urbanization. This scenario is illustrated in Figure 1A, where individuals with high intercept values for boldness are more likely to occur in more disturbed areas, leading to a positive among-individual effect of disturbance on behavior (i.e., $\beta_{\mathrm{A}}>0$, A for "among") and a zero within-individual effect (i.e., $\beta_{\mathrm{W}}=0, \mathrm{~W}$ for "within"). In the second scenario, individuals are instead randomly distributed over an urban gradient with respect to their intercept (i.e., behavioral type) while simultaneously not responding plastically to changes in urbanization. This scenario is illustrated in Figure 1B, showing a zero among- $\left(\beta_{\mathrm{A}}=0\right)$ and a zero within-individual effect $\left(\beta_{\mathrm{W}}=\right.$ $0)$. In the third scenario, individuals are both non-randomly distributed over an urban gradient with respect to their intercept while also responding plastically to changes in urbanization. This scenario is illustrated in Figure 1C, where individuals with high intercept values for boldness (i.e., bold behavioral types) are more likely to occur in more disturbed areas, leading to a nonzero among-individual effect of disturbance on behavior (i.e., $\beta_{\mathrm{A}}>0$ ), while individuals also alter their phenotype in response to changes in level of disturbance, leading to a nonzero within-individual effect (i.e., $\beta_{\mathrm{W}}>0$ ). In both scenarios 1 and 3 , importantly, the effect of non-random distributions of behavioral types causes a difference between the among- and within-individual effect of the environmental gradient (van de Pol and Verhulst, 2006) (specifically, $\beta_{\mathrm{W}}<\beta_{\mathrm{A}}$ in both examples). In the fourth scenario, individuals do not differ in intercept (i.e., all have the same behavioral type) but they do respond plastically to changes in urbanization. This scenario is illustrated in Figure 1D, where individuals up-regulate their boldness with increasing levels of disturbance (i.e., $\beta_{\mathrm{W}}>0$ ), and where amongindividual relationships between boldness and disturbance exist solely because each individual happens to experience a narrow range of environmental conditions, causing among-individual variance in the average level of disturbance experienced (i.e., $\left.\beta_{\mathrm{A}}>0\right)$. As a single mechanism causes variation at both levels, environmental effects on behavior do not differ between hierarchical levels (van de Pol and Verhulst, 2006) (i.e., $\beta_{\mathrm{A}}=\beta_{\mathrm{w}}$ in Figure 1D). In summary, our sketch of alternative scenarios clarifies that conclusions regarding non-random distributions of behavioral phenotypes over urban gradients warrants repeated observations of both behavior and environmental factors such that level-specific effects of urbanization on behavior can be statistically teased apart (Figure 1).

We investigated the mechanisms causing relationships between urbanization and behavior in great tits. We simultaneously and repeatedly quantified an individual's behavior and environment. That is, we repeatedly exposed the same individual to territorial intrusion experiments to measure aggressiveness (Araya-Ajoy and Dingemanse, 2014) and to flight initiation distance experiments to measure boldness (Blumstein, 2003), while quantifying aspects of urbanization during each test (numbers of pedestrians, cars and cyclists). This enabled us to statistically distinguish within-individual plasticity from patterns of non-random distributions of behavioral types (Figure 1). Human activity is known to alter behavioral phenotypes in animals (Fernandez-Juricic and Schroeder, 2003; Slabbekoorn and Peet, 2003). Based on previous literature (Brumm, 2004; Evans et al., 2010), we expected that birds would plastically up-regulate aggressiveness and down-regulate flight initiation distance with increasing levels of disturbance, and that more aggressive and bolder individuals would occur predominantly in areas with higher levels of disturbance (Figure 1C).

\section{METHODS}

\section{Study Site}

We studied an urban nest box population of great tits consisting of 157 nest boxes along urban-to-rural gradients in the city of Munich, Germany (48 $\left.8^{\circ} 6.45^{\prime \prime} \mathrm{N} 11^{\circ} 34^{\prime} 55.132^{\prime \prime} \mathrm{E}\right)$ during the breeding seasons of the years 2014 and 2015 (Sprau et al., 2016). Nest boxes were located in the entire city area of Munich $\left(20 \times 27 \mathrm{~km}^{2}\right)$ and covered a large range of human disturbance from highly disturbed habitats in the city center to relatively undisturbed habitats in sub-urban areas. All nest boxes were checked at least once per week from mid-March onwards and key fitness components quantified (e.g., lay date, clutch size, brood size, and number of fledged offspring). When the nestlings were 7-9 days old, parents were caught with a spring trap in the nest box, measured, and ringed if not previously captured.

\section{Experimental Protocol}

We quantified two behaviors both of which were assayed repeatedly for the same set of breeders: aggressiveness and boldness (measured as flight initiation distance; FID). Simulated territorial intrusions (i.e., aggression tests) were performed for all first broods found in our nest boxes by simultaneously presenting the male owner with a visual stimulus (a taxidermic mount of a male great tit) and an acoustic stimulus (a playback song) (as detailed in reference Araya-Ajoy and Dingemanse, 2014). In each year, each male was subjected to three aggression tests (between 7.30 and $15.00 \mathrm{~h}$ ) when its mate was in the egg-laying phase (1,3, and 5 days after its first egg was observed). The taxidermic mount was presented $1 \mathrm{~m}$ away from the subject's nest-box at $1.2 \mathrm{~m}$ height. We subsequently recorded the behavior of the subject for a period of $3 \mathrm{~min}$ after it had entered a 15$\mathrm{m}$ radius around the nest box. Details of the experimental set up, and assayed behaviors, are provided in reference ArayaAjoy and Dingemanse (2014). In short, an aggressive response was characterized by intensive alarm calling, approach to the stimulus, and, in the most extreme case, jumping and pecking of the cage that protected the mount. Here and elsewhere (ArayaAjoy et al., 2016; Araya-Ajoy and Dingemanse, 2017) we used the subject's minimum approach distance to the mount as a measure of aggressiveness because previous work implied that this behavior represents a reliable predictor of the intensity of aggression. Subjects that did not arrive within 10 min were scored 
as non-responsive, and those data were thus not taken forward for analyses (Araya-Ajoy and Dingemanse, 2014, 2017). We used six mounts and 11 playback song stimuli (recorded from German great tits populations; sampling frequency: $44.1 \mathrm{kHz}$; resolution: 16 bit). One mount and one song stimulus (broadcasted with a Ligno Xtatic V2 Digital Soundsystem) were randomly allocated to each test (following Araya-Ajoy and Dingemanse, 2014). Songs were played back at $85 \mathrm{~dB}$ (measured at one meter from the sound source (Brumm, 2004). One of nine observers performed the experiment at a distance of $15 \mathrm{~m}$. We performed 333 aggression tests with 107 unique males.

The occupants of each nest box were also subjected to three FID-tests during the nestling phase of their first brood (10, 12 and 14 days after the nestlings had hatched). FID-tests were conducted between 8:00 and 16:00 h. After identifying (by color ring combination) the focal individual as the male or female parent, FID was measured by walking at a constant speed toward a bird from a starting distance of $15 \mathrm{~m}$ away from the nest box (Blumstein, 2003). We used a laser distance meter (Bosch PLR 25) to quantify distance to the mount during the aggression tests, and start and flight initiation distance. Overall, we performed 308 flight initiation tests, on 59 females and 54 males. Note that because of nest failure prior to the onset of the FID-tests, the number of FID-tests is lower than the number of the aggression tests. Five and four individuals were assayed, respectively, for aggressiveness and flight initiation distance in both years of study. This study was carried out in accordance with the ethical guidelines of the Tierschutzgesetz (TierSchG, German animal protection law), and approved by the Regierung Oberbayern (55.2-1-54-2532.2-7-07).

The level of urbanization at each nest box was quantified by measuring human activity (the number of pedestrians, cyclists and cars, Table S1). Human activity was measured within a range of $15 \mathrm{~m}$ from the nest box for 2 min following each behavioral assay (detailed above).

\section{Statistical Analyses}

We performed a principal component analysis (PCA) with varimax rotation ("prcomp" function of Package "stats" version 3.1.27 of $\mathrm{R}$ version 3.1.2) to ask whether our indexes of human activity (number of pedestrians, bikes and cars) could be summarized into a single axis (principal component) representing an urban gradient (Table $\mathrm{S} 1$ ).

We fitted univariate mixed-effect models to simultaneously estimate sources of variation in behavior within and among individuals (Dingemanse and Dochtermann, 2013) ("lmer" function of Package "lme4"version 1.1-10 of R). We investigated sources of variation in each of the two focal behaviors (aggressiveness and flight initiation distance) separately. Random intercepts were included for subject and observer identity, enabling us to partition the total variance into variance attributable to individual, observer, and within-individualwithin-observer residual. Start distance (covariate: only for flight initiation distance; meter), year (factor: 2014 vs. 2015), time of day (factor: morning vs. afternoon trial), and test sequence (covariate; within-individual test-day number; first vs. second vs. third test day) were fitted as fixed effects. The PCA resulted in two components (PC1 and PC2) describing two orthogonal axes of human activity. As detailed in the Introduction (Figure 1), we considered that environmental effects on behavior could vary within and among individuals (van de Pol and Wright, 2009). Specifically, a within-individual effect of the environment on behavior represents evidence for within-individual phenotypic plasticity, while the difference between among- and withinindividual effects represents statistical evidence for non-random distributions of behavioral types over environments (van de Pol and Wright, 2009; Dingemanse and Dochtermann, 2013). We thus calculated (1) each individual's average value $\left(\bar{x}_{j}\right)$ for each of the two environmental variables (PC1 and PC2) as well as (2) each observation's deviation of these individual average values $\left(x_{i j}-\bar{x}_{j}\right)$ effects, and fitted both as part of the statistical model detailed above (van de Pol and Verhulst, 2006; van de Pol and Wright, 2009). We then reformulated the model to test whether the effect of the focal environmental axis differed between the within- and among-individual levels. Therefore, instead of fitting $\bar{x}_{j}$ and $\left(x_{i j}-\bar{x}_{j}\right)$ and each individual's average value $\left(\bar{x}_{j}\right)$ instead, such that the former estimated the within-individual effect $\left(\beta_{W}\right)$ (see formulae 3 in van de Pol and Wright, 2009). This enabled us to statistically assess the evidence for non-random distributions of behavioral types over environments (which would be the case provided that $\left.\left(\beta_{A}-\beta_{W}\right) \neq 0\right)$. We note that the within-subject centering approach used here has been criticized because values of $\bar{x}_{j}$ are estimated with error, which causes estimates of the among-individual slope $\left(\beta_{A}\right)$ to be biased toward the withinindividual slope $\left(\beta_{W}\right)$ in datasets with a low numbers of repeats per individual (Ludtke et al., 2008), such as ours. This means that differences $(\Delta)$ between among- and within-individual effects (i.e., $\beta_{A}-\beta_{W}$ ), as well as associated levels of significance, represent conservative estimates. We assumed a Gaussian error distribution for aggression and boldness, which was confirmed by visual inspection of model residuals. All covariates were further centered on their mean value (Kreft et al., 1995). For each specified relationship, we calculated the parameter estimate with its associated 95\% credible interval (calculated using the function "quantile"; Package "stats"version 3.1.2 in R). Credible intervals not including zero indicate statistical significance (i.e., $p<0.05$ ) in the frequentist's sense.

\section{RESULTS}

\section{Axes of Environmental Variation}

PCA applied to summarize variation in the number of pedestrians, cyclists, and cars, resulted in two significant principle components (PCs) that jointly explained $81 \%$ of the variance (Table S2). PC1 (Eigenvalue: 1.40; explained variance: 47\%) loaded negatively on the number of bikes, cars, and pedestrians (Table S1); high values of PC1 were thus indicative of lower levels of human activity in general. PC2 (Eigenvalue: 1.04; explained variance: $35 \%)$ loaded negatively on the number of cars $(-0.76)$ but positively on the number of pedestrians (0.65), and thus seemed to differentiate between streets differing in the primary means of transportation (e.g., larger streets suitable for cars vs. smaller streets suitable for pedestrians). 


\section{Phenotypic Plasticity and Non-random Distributions of Behavioral Types}

Our analyses of the sources of variation in behavior, which focussed on the simultaneous estimation of within-individual $\left(\beta_{W}\right)$ and among-individual $\left(\beta_{A}\right)$ effects of environmental variables related to urbanization (Figure 1), demonstrated that individuals did not plastically adjust their aggressiveness nor their flight initiation distance (FID) in response to withinindividual-among-day variation in PC1 or PC2 (Table 1). All models controlled for variation induced by aspects of the experimental design (starting distance, test sequence, time of day, and year), which were generally not of major importance (Table 1).

As a next step, we re-parameterised our models to directly estimate the difference $(\Delta)$ between the among- and withinindividual effects $\left(\beta_{A}-\beta_{W}\right)$ of each focal gradient as a test for non-random distributions of behavioral types (van de Pol and Verhulst, 2006; van de Pol and Wright, 2009). This analysis produced strong evidence for non-random distributions of behavioral types with respect to FID because the difference $(\Delta)$ in effect of PC2 among- vs. within-individuals was associated with $95 \%$ CIs that did not overlap zero (mode: 0.28; 95\% CIs: $0.03,0.56$; Table 1). The analysis implied that individuals that allowed observers to approach closer (i.e., "bolder" birds) were overrepresented in areas with more cars and fewer pedestrians, whereas "shyer" birds were more likely found in areas with fewer cars and more pedestrians (Figure 2). By contrast, there was no strong evidence for nonrandom distributions of aggressiveness types as the 95\% CIs overlapped zero for all tested differences $(\Delta)$ between the within- and among-individual levels for aggressive behavior (Table 1). Notably, there was some support for non-random distributions of aggressiveness types with respect to PC1 as the CIs associated with the difference $(\Delta)$ between levels for this gradient slightly overlapped zero (95\% CIs: -0.001 , 0.38) (Table 1), suggesting that aggressive types were perhaps overrepresented in areas with more cyclists, cars, and pedestrians (Table 1).

\section{DISCUSSION}

Our study revealed that behavioral types, with respect to flight initiation distance, were non-randomly distributed over an urban gradient while individuals did not plastically adjust their behavior in response to changes in urban gradients experienced across repeated observations (days). Bolder birds (i.e., birds that could be approached by humans closely) were overrepresented in areas with more cars and fewer pedestrians, whereas shyer birds were more likely found in areas with fewer cars and more pedestrians (Figure 2). These findings imply that associations between behavior and urban gradients vary across hierarchical levels, in this case within and among individuals, and that meaningful conclusions regarding non-random distributions of "personality" types over urban environments thus require repeated measures study designs and variance partitioning approaches, as applied in this study.
TABLE 1 | Sources of variation in boldness and aggressiveness.

\begin{tabular}{|c|c|c|c|c|}
\hline \multirow[b]{2}{*}{ Fixed effects } & \multicolumn{2}{|c|}{$\begin{array}{l}\text { Boldness (Flight initiation } \\
\text { distance) }\end{array}$} & \multicolumn{2}{|c|}{$\begin{array}{l}\text { Aggressiveness (Minimal } \\
\text { approach distance) }\end{array}$} \\
\hline & Estimate & $95 \%$ Cls & Estimate & $95 \%$ Cls \\
\hline Intercept & -0.57 & $-0.93,-0.19$ & 0.05 & $-0.22,0.34$ \\
\hline \multicolumn{5}{|l|}{ PC1 } \\
\hline Within individuals & -0.14 & $-0.34,0.06$ & -0.06 & $-0.23,0.13$ \\
\hline Among individuals & 0.07 & $-0.08,0.22$ & 0.13 & $-0.02,0.27$ \\
\hline$\Delta$ (Among-within) & 0.19 & $-0.01,0.42$ & 0.19 & $-0.001,0.38$ \\
\hline \multicolumn{5}{|l|}{ PC2 } \\
\hline Within individuals & -0.12 & $-0.41,0.16$ & 0.12 & $-0.23,0.46$ \\
\hline Among individuals & 0.17 & $0.04,0.30$ & -0.05 & $-0.22,0.10$ \\
\hline$\Delta$ (Among - within) & 0.28 & $0.03,0.56$ & -0.17 & $-0.55,0.17$ \\
\hline Start distance & -0.11 & $-0.22,0.01$ & n.a. & n.a. \\
\hline Year & -0.13 & $-0.57,0.30$ & 0.12 & $-0.15,0.38$ \\
\hline \multicolumn{5}{|c|}{ SEQUENCE (FIRST TEST AS REFERENCE) } \\
\hline Second test & 0.25 & $-0.04,0.54$ & -0.05 & $-0.37,0.23$ \\
\hline Third test & 0.23 & $-0.07,0.56$ & -0.08 & $-0.4,0.21$ \\
\hline Time of day & -0.09 & $-0.26,0.07$ & 0.04 & $-0.09,0.17$ \\
\hline Random effects & $\sigma^{2}$ & $95 \%$ Cls & $\sigma^{2}$ & $95 \%$ Cls \\
\hline Individual & 0.013 & $0.01,0.02$ & 0.017 & $0.01,0.02$ \\
\hline Observer & 0.20 & $0.12,0.42$ & 0.04 & $0.02,0.08$ \\
\hline Residual & 0.65 & $0.56,0.84$ & 0.91 & $0.80,1.14$ \\
\hline
\end{tabular}

We test here for within- and among individual effects of cyclists, cars, and pedestrians (summarized in PC1 and PC2, see Table S1). All models control for variation induced by various aspects of the experimental design (starting distance, test sequence, time of day, and year) and included random intercepts for subject individual and observer identity. We also present the difference $(\Delta)$ between among and within-individual effects derived from the same statistical model reformulated following reference (van de Pol and Wright, 2009). Parameter estimates and are provided with 95\% credible intervals (Cls).

Human activity is known to affect behavior and abundance of animals (Gill et al., 1996; Fernandez-Juricic, 2000); there is considerable evidence that birds living in highly disturbed areas are more tolerant of humans than their conspecifics living in less disturbed areas (Moller, 2008; Evans et al., 2010; Scales et al., 2011; Clucas and Marzluff, 2012). In a similar vein, recent studies have shown that birds in more disturbed environments may display higher levels of territorial and defensive behaviors (Cilento and Jones, 1999; Evans et al., 2010; Fokidis et al., 2011; Scales et al., 2011). Most of these studies, however, have solely focused on differences in behavioral phenotypes at the population level. Researchers have only recently begun to assess individual variation in the context of urban ecology (Miranda et al., 2013). In this study we shed new light on relationships between urbanization and behavioral phenotypes by partitioning variation in behavioral phenotypes into within- and amongindividual components; this enabled us to investigate the relative roles of distinct mechanisms causing such associations. The applied approach allowed us to simultaneously assess whether individuals responded plastically to urban gradients and whether behavioral types were non-randomly distributed over urban gradients (Figure 1). Our findings reveal that behavioral types were indeed non-randomly distributed along a key axis of 


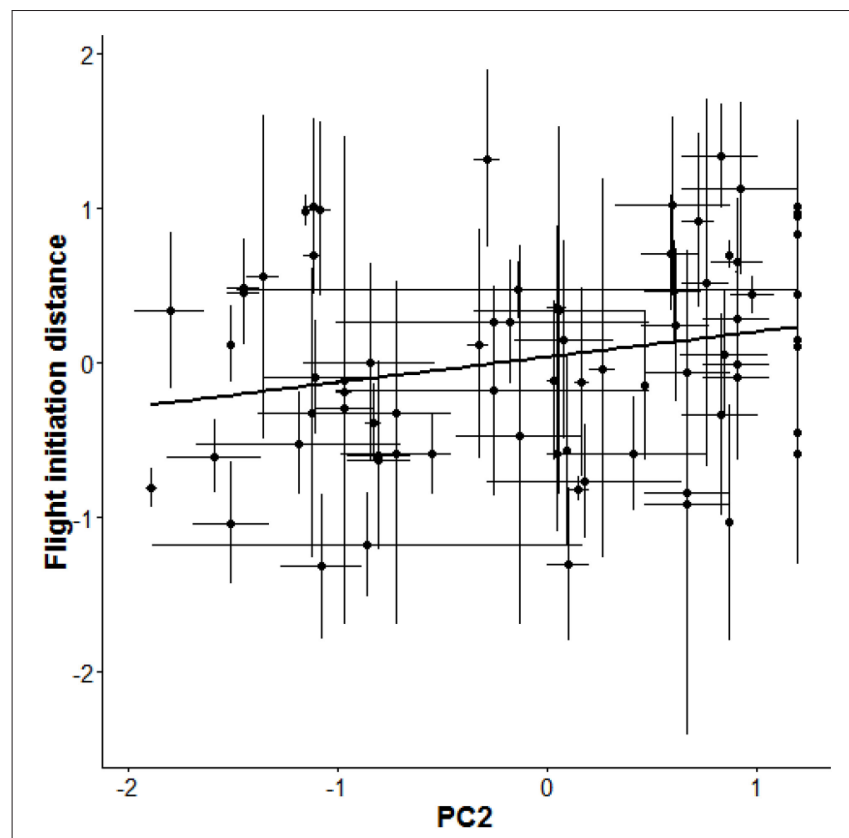

FIGURE 2 | Among-individual variation in flight initiation distance (FID) as a function of the compound environmental gradient PC2 that combines number of pedestrians and cars (more positive loadings represent more pedestrians and lower loadings more cars). As individuals do not plastically respond to changes in PC2 (Table 1), the relationship implies a non-random distribution of individual-level behavioral type along this urban gradient. The thick black line presents the regression line between FID and PC2 based on the fitted GLMM (Table 1). Shown are average scores \pm SE.

urbanization (Figure 2): bolder individuals, i.e., birds that could be approached by humans closely, were predominantly found in areas with more cars, whereas shyer individuals were found more often in areas with more pedestrians. The documented effect might be explained by sensory constraints caused by traffic noise. We tested this post-hoc explanation by analyzing noise measurements that were taken during each test (detailed in the Supplementary Material), which demonstrated that noise neither affected FID nor aggression (Table S3).

Non-random distributions of behavioral types along environmental gradients have previously been documented in eastern chipmunks (Tamias striatus) where more explorative and docile individuals occupy habitats that experience the highest rates of human disturbance (Martin and Réale, 2008). Accordingly, bold animals might have an innately higher disturbance tolerance level than shy individuals. In urban environments, cars usually impose high risks as evident from high numbers of road kills (Spellerberg, 1998; Benitez-Lopez et al., 2010). In contrast, pedestrians may impose lower disturbance levels because birds quickly habituate to humans. Because bold individuals are often considered to cope better with risky situations (Smith and Blumstein, 2008), bold phenotypes in our study may be selected for in high-traffic environments because bolder individuals are more successful in colonizing such environments. Alternatively, bold phenotypes might be outcompeted by shy phenotypes for preferred types of territories and hence settle in these types of areas. It is also possible that prolonged exposure to particular environments permanently affects an individual's behavioral type, resulting in birds becoming bolder in high-traffic environments by means of developmental or other forms of plasticity with permanent effects. Experimental tests are therefore now required to address whether the non-random distributions of behavioral phenotypes documented in our study were caused by nonrandom settlement, habitat- and type-specific survival, or irreversible plasticity in response to long-term exposure to urban environmental effects. Similarly, we studied a very specific component of the urban environment, focusing on human traffic. Whether the relationships between behavior and aspects of urbanization shown in this paper apply generally to other components of urbanization remains to be evaluated by future studies.

Surprisingly, great tits did not show any sign of a plastic response to day-to-day variation in urban environmental gradients. It is possible that exposure to high levels of human disturbance for prolonged periods triggers habituation (see above) and consequently reduces short-term plastic responses. Such effects may suggest that birds experience only minor fluctuations in environmental conditions, and that each bird's characteristic level of urbanization is relatively stable. In urban environments, such a scenario seems unlikely as the numbers of cars, pedestrians and cyclists, in fact, varied substantially, for instance, between workdays and weekends. Temporal variation between workdays and weekends has in fact previously been shown to cause plastic adjustments in other behaviors (Brumm, 2004). We therefore conclude that phenotypic adjustments to day-to-day variation in human disturbance might well differ between behavioral traits, perhaps because the costs or limits associated with phenotypic plasticity are trait-specific (DeWitt et al., 1998; Auld et al., 2010). Our recent studies on aggressiveness, for example, demonstrated that this particular behavior (which birds did not plastically adjust to changes in human disturbance; Table 1) is also not plastically adjusted to population density (Araya-Ajoy and Dingemanse, 2017) or perceived predation risk (Abbey-Lee et al., 2016). Overall, the lack of evidence for within-individual plasticity suggests that its role in urban ecology may be more modest than previously anticipated (Lowry et al., 2013). At the same time, urbanization seems to drive non-random distributions of behavioral types via mechanisms yet to be revealed. Our study thereby demonstrates the importance of partitioning behavioral variation across hierarchical levels (Han et al., 2016; Moirón et al., 2016; Nicolaus et al., 2016), both in urban and other behavioral ecological studies, and the novel insights that may be gained by doing so.

In conclusion, we showed for great tits breeding in the city that behavioral types were non-randomly distributed over an urban environmental gradient. Based on these findings, future research should investigate whether non-random distribution of types is caused by selective appearance (i.e., differential settlement), selective disappearance (i.e., natural selection) or urbanizationrelated behavioral modification (i.e., developmental or other forms of irreversible plasticity). 


\section{AUTHOR CONTRIBUTIONS}

PS and ND conceived and designed the study, conceived the statistical approach, and wrote the manuscript

\section{ACKNOWLEDGMENTS}

We thank past and current members, as well as field assistants and students of the Behavioral Ecology Group (LudwigMaximilians University Munich) and the research group Evolutionary Ecology of Variation (Max Planck Institute for Ornithology), as well as all citizen scientists for input, discussion,

\section{REFERENCES}

Abbey-Lee, R. N., Kaiser, A., Mouchet, A., and Dingemanse, N. J. (2016). Immediate and carry-over effects of perceived predation risk on communication behavior in wild birds. Behav. Ecol. 27, 708-716. doi: 10.1093/beheco/arv210

Araya-Ajoy, Y. G., and Dingemanse, N. J. (2017). Repeatability, heritability, and age-dependence of seasonal plasticity in aggressiveness in a wild passerine bird. J. Anim. Ecol. 86, 227-238. doi: 10.1111/1365-2656.12621

Araya-Ajoy, Y. G., and Dingemanse, N. J. (2014). Characterizing behavioural 'characters': an evolutionary framework. Proc. R. Soc. B Biol. Sci. 281:20132645. doi: $10.1098 /$ rspb.2013.2645

Araya-Ajoy, Y. G., Kuhn, S., Mathot, K. J., Mouchet, A., Mutzel, A., Nicolaus, M., et al. (2016). Sources of (co)variation in alternative siring routes available to male great tits (Parus major). Evolution 70, 2308-2321. doi: 10.1111/evo. 13024

Auld, J. R., Agrawal, A. A., and Relyea, R. A. (2010). Re-evaluating the costs and limits of adaptive phenotypic plasticity. Proc. R. Soc. Lond. B Biol. Sci. 277, 503-511. doi: 10.1098/rspb.2009.1355

Beissinger, S. R., and Osborne, D. R. (1982). Effects of urbanization on avian community organisation. Condor 84, 75-83. doi: 10.2307/1367825

Bell, A. M., Hankison, S. J., and Laskowski, K. L. (2009). The repeatability of behaviour: a meta-analysis. Anim. Behav. 77, 771-783. doi: 10.1016/j.anbehav.2008.12.022

Benitez-Lopez, A., Alkemade, R., and Verweij, P. A. (2010). The impacts of roads and other infrastructure on mammal and bird populations: a meta-analysis. Biol. Conserv. 143, 1307-1316. doi: 10.1016/j.biocon.2010.02.009

Blumstein, D. T. (2003). Flight-initiation distance in birds is dependent on intruder starting distance. J. Wildlife Manage. 67, 852-857. doi: 10.2307/3802692

Bouchard, J., Goodyer, W., and Lefebvre, L. (2007). Social learning and innovation are positively correlated in pigeons (Columba livia). Anim. Cogn. 10, 259-266. doi: 10.1007/s10071-006-0064-1

Brumm, H. (2004). The impact of environmental noise on song amplitude in a territorial bird. J. Anim. Ecol. 73, 434-440. doi: 10.1111/j.0021-8790.2004.00814.x

Chamberlain, D. E., Cannon, A. R., Toms, M. P., Leech, D. I., Hatchwell, B. J., and Gaston, K. J. (2009). Avian productivity in urban landscapes: a review and meta-analysis. Ibis 151, 1-18. doi: 10.1111/j.1474-919X.2008.00899.x

Cilento, N. J., and Jones, D. N. (1999). Aggression by Australian magpies Gymnorhina tibicen toward human intruders. Emu 99, 85-90. doi: 10.1071/MU99011

Clucas, B., and Marzluff, J. M. (2012). Attitudes and actions toward birds in urban areas: human cultural differences influence bird behavior. Auk 129, 8-16. doi: 10.1525/auk.2011.11121

DeWitt, T. J., Sih, A., and Wilson, D. S. (1998). Costs and limits of phenotypic plasticity. Trends Ecol. Evol. 13, 77-81. doi: 10.1016/S0169-5347(97)01274-3

Dingemanse, N. J., and Dochtermann, N. A. (2013). Quantifying individual variation in behaviour: mixed-effect modelling approaches. J. Anim. Ecol. 82, 39-54. doi: 10.1111/1365-2656.12013 and/or help with data collection. Werner Schimmel and Dietfried Molter are acknowledged for technical support and Petri Niemelä for stimulating discussion. PS was funded by the Deutsche Forschungsgemeinschaft (SP 1450/3-1) and ND by the Max Planck Society.

\section{SUPPLEMENTARY MATERIAL}

The Supplementary Material for this article can be found online at: http://journal.frontiersin.org/article/10.3389/fevo. 2017.00092/full\#supplementary-material

Dingemanse, N. J., Kazem, A. J. N., Reale, D., and Wright, J. (2010) Behavioural reaction norms: animal personality meets individual plasticity. Trends Ecol. Evol. 25, 81-89. doi: 10.1016/j.tree.2009. 07.013

Evans, J., Boudreau, K., and Hyman, J. (2010). Behavioural syndromes in urban and rural populations of Song Sparrows. Ethology 116, 588-595. doi: 10.1111/j.1439-0310.2010.01771.x

Fernandez-Juricic, E. (2000). Local and regional effects of pedestrians on forest birds in a fragmented landscape. Condor 102, 247-255. doi: 10.1650/00105422(2000)102[0247:LAREOP]2.0.CO;2

Fernandez-Juricic, E., and Schroeder, N. (2003). Do variations in scanning behavior affect tolerance to human disturbance? Appl. Anim. Behav. Sci. 84, 219-234. doi: 10.1016/j.applanim.2003. 08.004

Fokidis, H. B., Orchinik, M., and Deviche, P. (2011). Context-specific territorial behavior in urban birds: no evidence for involvement of testosterone or corticosterone. Horm. Behav. 59, 133-143. doi: 10.1016/j.yhbeh.2010. 11.002

Garamszegi, L. Z., Markó, G., and Herczeg, G. (2012). A meta-analysis of correlated behaviours with implications for behavioural syndromes: mean effect size, publication bias, phylogenetic effects and the role of mediator variables. Evol. Ecol. 26, 1213-1235. doi: 10.1007/s10682-012-9589-8

Gill, J. A., Sutherland, W. J., and Watkinson, A. R. (1996). A method to quantify the effects of human disturbance on animal populations. J. Appl. Ecol. 33, 786-792. doi: $10.2307 / 2404948$

Han, C. S., Jäger, H. Y., and Dingemanse, N. J. (2016). Individuality in nutritional preferences: a multi-level approach in field crickets. Sci. Rep. 6:29071. doi: $10.1038 /$ srep29071

Holtmann, B., Lagisz, M., and Nakagawa, S. (2017). Metabolic rates, and not hormone levels, are a likely mediator of between-individual differences in behaviour: a meta-analysis. Funct. Ecol. 31, 685-696. doi: 10.1111/1365-2435.12779

Kreft, I. G. G., Deleeuw, J., and Aiken, L. S. (1995). The effect of different forms of centering in hierarchical linear-models. Multiv. Behav. Res. 30, 1-21. doi: $10.1207 /$ s15327906mbr3001_1

Lefebvre, L. (1995). The opening of milk bottles by birds - evidence for accelerating learning rates, but agains the wave-of-advance model of cultural transmission. Behav. Processes 34, 43-53. doi: 10.1016/0376-6357(94)00051-H

Levey, D. J., Londono, G. A., Ungvari-Martin, J., Hiersoux, M. R., Jankowski, J. E., Poulsen, J. R., et al. (2009). Urban mockingbirds quickly learn to identify individual humans. Proc. Natl. Acad. Sci. U.S.A. 106, 8959-8962. doi: $10.1073 /$ pnas.0811422106

Lowry, H., Lill, A., and Wong, B. B. M. (2013). Behavioural responses of wildlife to urban environments. Biol. Rev. 88, 537-549. doi: 10.1111/brv. 12012

Ludtke, O., Marsh, H. W., Robitzsch, A., Trautwein, U., Asparouhov, T., and Muthen, B. (2008). The multilevel latent covariate model: a new, more reliable approach to group-level effects in contextual studies. Psychol. Methods 13, 203-229. doi: 10.1037/a0012869 
Martin, J. G. A., and Réale, D. (2008). Temperament, risk assessment and habituation to novelty in eastern chipmunks, Tamias striatus. Anim. Behav. 75, 309-318. doi: 10.1016/j.anbehav.2007.05.026

Marzluff, J. M. (2008). Urban Ecology: An International Perspective on the Interaction between Humans and Nature. New York, NY: Springer.

Miranda, A. C., Schielzeth, H., Sonntag, T., and Partecke, J. (2013). Urbanization and its effects on personality traits: a result of microevolution or phenotypic plasticity? Glob. Chang. Biol. 19, 2634-2644. doi: 10.1111/gcb.12258

Moirón, M., Mathot, K. J., and Dingemanse, N. J. (2016). A multi-level approach to quantify speed-accuracy trade-offs in great tits (Parus major). Behav. Ecol. 27, 1539-1546. doi: 10.1093/beheco/arw077

Moller, A. P. (2008). Flight distance of urban birds, predation, and selection for urban life. Behav. Ecol. Sociobiol. 63, 63-75. doi: 10.1007/s00265-008-0636-y

Nicolaus, M., Piault, R., Ubels, R., Tinbergen, J. M., and Dingemanse, N. J. (2016). The correlation between colouration and exploration behaviour varies across hierarchical levels in a wild passerine bird. J. Evol. Biol. 29, 1780-1792. doi: $10.1111 /$ jeb.12907

Nussey, D., Wilson, A., and Brommer, J. (2007). The evolutionary ecology of individual phenotypic plasticity in wild populations. J. Evol. Biol. 20, 831-844. doi: 10.1111/j.1420-9101.2007.01300.x

Réale, D., Reader, S. M., Sol, D., McDougall, P. T., and Dingemanse, N. J. (2007). Integrating animal temperament within ecology and evolution. Biol. Rev. 82, 291-318. doi: 10.1111/j.1469-185X.2007.00010.x

Scales, J., Hyman, J., and Hughes, M. (2011). Behavioral syndromes break down in urban song sparrow populations. Ethology 117, 887-895. doi: 10.1111/j.1439-0310.2011.01943.x

Seferta, A., Guay, P. J., Marzinotto, E., and Lefebvre, L. (2001). Learning differences between feral pigeons and zenaida doves: the role of neophobia and human proximity. Ethology 107, 281-293. doi: 10.1046/j.1439-0310.2001. 00658.x

Sih, A., Ferrari, M. C. O., and Harris, D. J. (2011). Evolution and behavioural responses to human-induced rapid environmental change. Evol. Appl. 4, 367-387. doi: 10.1111/j.1752-4571.2010.00166.x

Slabbekoorn, H., and den Boer-Visser, A. (2006). Cities change the songs of birds. Curr. Biol. 16, 2326-2331. doi: 10.1016/j.cub.2006.10.008
Slabbekoorn, H., and Peet, M. (2003). Ecology: birds sing at a higher pitch in urban noise - Great tits hit the high notes to ensure that their mating calls are heard above the city's din. Nature 424, 267-267. doi: 10.1038/42 $4267 \mathrm{a}$

Smith, B. R., and Blumstein, D. T. (2008). Fitness consequences of personality: a meta-analysis. Behav. Ecol. 19, 448-455. doi: 10.1093/beheco/arm144

Spellerberg, I. F. (1998). Ecological effects of roads and traffic: a literature review. Glob. Ecol. Biogeogr. 7, 317-333. doi: 10.1046/j.1466-822x.1998.00308.x

Sprau, P., Mouchet, A., and Dingemanse, N. J. (2016). Multidimensional environmental predictors of variation in avian forest and city life histories. Behav. Ecol. 28, 59-68. https://doi.org/10.1093/beheco/arw130

Stearns, S. C. (1989). The evolutionary significance of phenotypic plasticity. Bioscience 39, 436-445. doi: 10.2307/1311135

United Nations, Department of Economic and Social Affairs, and Population Division (2017). World Population Prospects. New York, NY: United Nations.

van de Pol, M., and Verhulst, S. (2006). Age-dependent traits: a new statistical model to separate within- and between-individual effects. Am. Nat. 167, 766-773. doi: 10.1086/503331

van de Pol, M., and Wright, J. (2009). A simple method for distinguishing withinversus between-subject effects using mixed models. Anim. Behav. 77, 753-758. doi: 10.1016/j.anbehav.2008.11.006

Wilson, D. S., Clark, A. B., Coleman, K., and Dearstyne, T. (1994). Shyness and boldness in humans and other animals. Trends Ecol. Evol. 9, 442-446. doi: 10.1016/0169-5347(94)90134-1

Conflict of Interest Statement: The authors declare that the research was conducted in the absence of any commercial or financial relationships that could be construed as a potential conflict of interest.

Copyright (C) 2017 Sprau and Dingemanse. This is an open-access article distributed under the terms of the Creative Commons Attribution License (CC BY). The use, distribution or reproduction in other forums is permitted, provided the original author(s) or licensor are credited and that the original publication in this journal is cited, in accordance with accepted academic practice. No use, distribution or reproduction is permitted which does not comply with these terms. 